Acta Crystallographica Section E

Structure Reports

Online

ISSN 1600-5368
Werner H. Baur

Department of Geophysical Sciences, University of Chicago, Chicago, Illinois 60637, USA

Correspondence e-mail:

wbaur@midway.uchicago.edu

\title{
Zinc(II) sulfate tetrahydrate and magnesium sulfate tetrahydrate. Addendum
}

The crystal structure of zinc(II) sulfate tetrahydrate, $\left[\mathrm{Zn}\left(\mathrm{SO}_{4}\right)\left(\mathrm{H}_{2} \mathrm{O}\right)_{4}\right]_{2}$, has recently been determined by Blake, Cooke, Hubberstey \& Sampson [Acta Cryst. (2001), E57, i109i111]. Additional information on structure determinations of similar compounds is given.

\section{Comment}

The crystal structure of zinc (II) sulfate tetrahydrate has recently been determined by Blake et al. (2001). The structure contains dimers in the form of eight-membered rings formed by two sulfate coordination tetrahedra and two coordination octahedra around zinc, where the ligands of zinc are two sulfate $\mathrm{O}$ atoms and four water molecules (see Fig. 1 in Blake et al., 2001). These rings are hydrogen bonded to each other via hydrogen bonds emanating from the water molecules. This type of dimer is said to be very rare for zinc(II), but dimers of similarly sized divalent cations are not so rare. In fact, the crystal structures of magnesium sulfate tetrahydrate and iron(II) sulfate tetrahydrate have been determined (Baur, 1962) and are strictly isostructural with the zinc(II) sulfate tetrahydrate, as is the deuterated cobalt(II) sulfate tetrahydrate (Kellersohn, 1992). The positions of the heavy atoms $\mathrm{Mg}, \mathrm{S}$ and $\mathrm{O}$ in the respective magnesium and zinc compounds deviate on average only by $0.06 \AA$ from each other. The signs of the $y$ coordinates of the atoms in the zinc compound have to be changed in order to make the similarity obvious. On the basis of a determination of the unit-cell constants, the crystal structure of the corresponding manganese(II) sulfate tetrahydrate must also be isostructural (Baur, 1962). The crystal structure of the magnesium sulfate tetrahydrate was determined by single-crystal neutron diffraction (Baur, 1964). The positions of the $\mathrm{H}$ atoms of three of the crystallographically distinct water molecules agree as well in the two structures as can be expected from a comparison of an X-ray and a neutron diffraction determination; they differ on average by $0.26 \AA$ from each other. Atom $\mathrm{O} 2$ in the zinc compound corresponds to atom $\mathrm{OW} 2$ in the magnesium compound. The two $\mathrm{H}$ atoms of water molecule $\mathrm{O} 2$ deviate by 0.6 and $1.0 \AA$ from the $\mathrm{H}$ atoms attached to water molecule OW2. Either the X-ray crystal structure determination is so much less precise or else the positions of the $\mathrm{H}$ atoms differ considerably between the magnesium and zinc compounds. This is the water molecule in which only one of the $\mathrm{H}$ atoms is engaged in a relatively strong hydrogen bond, while the other is not hydrogen bonded (Baur, 1965). The situation might be similar in the zinc compound. The positions of the $\mathrm{H}$ atoms in the magnesium sulfate tetrahydrate and the cobalt(II) sulfate tetrahydrate agree well with each other. 


\section{References}

Baur, W. H. (1962). Acta Cryst. 15, 815-826.

Baur, W. H. (1964). Acta Cryst. 17, 863-869.
Baur, W. H. (1965). Acta Cryst. 19, 909-916.

Blake, A. J., Cooke, P. A., Hubberstey, P. \& Sampson, C. L. (2001). Acta Cryst. E57, i109-i111.

Kellersohn, T. (1992). Acta Cryst. C48, 776-779. 


\section{supporting information}

Acta Cryst. (2002). E58, e9-e10 [https://doi.org/10.1107/S1600536802002192]

Zinc(II) sulfate tetrahydrate and magnesium sulfate tetrahydrate. Addendum

Werner H. Baur 